\title{
Qualitative Evaluation of Cross-Layer Approaches in Wireless Sensor Networks
}

\author{
Waltenegus Dargie \\ Chair for Computer Networks, Faculty of Computer Science \\ Technical University of Dresden \\ 01062 Dresden, Germany \\ Email: waltenegus.dargie@tu-dresden.de
}

\begin{abstract}
This paper provides a qualitative analysis of cross-layer approaches in wireless sensor networks. It begins by presenting two approaches in cross-layer design. The first one focuses on the existence of an auxiliary communication link between the layers - here the OSI layered-approach is being implied - in order to support the exchange of information which is useful for adaptation. The approach itself leaves the layered architecture intact. The second approach takes the OSI layer as a reference, but merges two or more layers together or defines an entirely new dimension to support efficient communication. Therefore, the newly formed architecture requires, in part or wholly, new suit of protocols which may not fit into the layered architecture. The flexibility and performance of the two approaches is compared by investigating some proposed network architectures.
\end{abstract}

\section{INTRODUCTION}

A cross-layer architectural design has been the subject of research in wireless sensor networks. Unlike general purpose computer networks, these networks are primarily designed with a specific sensing task in mind. Moreover, energy is a prime concern, since the nodes operate with exhaustible batteries; and the quality of data and the network's lifetime depend on the power consumption of the network. Naturally, these two create a tight dependency between several aspects of communications and networking. For example, most contention-based medium access control protocols adjust their contention window and duty cycle according to node density and expected lifetime [1]. Likewise, routing and self-organization protocols often require knowledge of congestion and node density as well as some non-functional aspects such as end-to-end delay requirements by the application. In fact, according to [2], almost all proposed energy-efficient communication protocols can be regarded as cross-layer.

Whereas the aim of cross-layer design is clear, there is no consensus in the literature as to its meaning [3].
For example, Srivastava and Motani [2] provide six different types of cross-layer design strategies: (a) upward information flow, (b) downward information flow, (c) back-and-forth information flow, (d) merging of adjacent layers, (e) introduction of an intermediate layer, and (f) vertical calibration. All of these definitions take the OSI reference framework into consideration. Except (d), the strategies are based on information exchange between two or more layers, adjacent or otherwise.

It is useful to distinguish between the existence of information links between two or more distinct layers (inter-layer communication) and the absence of a clear boundary between the layers (intra-layer communication). The inter-layer approaches are difficult to distinguish from the conventional layered architecture, because, they, too, support modularization and the protocols at different layers can be developed independently. In the latter case, there is a tight interdependence between multiple layers (and a significant reduction of communication overheads), such that the modification or replacement of one protocol without the modification or entirely replacement of some protocols or protocol parameters elsewhere is not possible.

This paper attempts to examine to which of these approaches proposed cross-layer architectures in wireless sensor network belong. The understanding will be useful for protocol developers and performance analysts. It should be noted that, architectures that are based on intra-layer communications are mostly energy-efficient, but costly to develop. In contrast, architectures that are based on inter-layer communications are flexible and reusable [4], but not energy-efficient.

The remaining part of this paper is organized as follows: In section III, example inter-layer approaches are presented. In section III], example intra-layer approaches are presented. Finally, in sec IV] a comparison discussion is given. 


\section{INTER-LAYER DESIGN}

In this section, three inter-layer architectures will be examined. These layers define physical, link, network and application layers; and create auxiliary links between the layers to enable information flow pertaining to the network status so that protocol parameter tuning can be made. It will be demonstrated that because of the need for multi-hop communications in wireless sensor networks, there is a tight dependency between the network and the link layer.

\section{A. Energy Efficient Scheduling (EES)}

The medium access control problem in wireless sensor networks has been extensively investigated for two essential reasons: (1) The problem of collision and the associated energy cost of packet retransmission; and (2) the need for self-organization (without an energyefficient link layer protocol, self-organization cannot be supported). As a result, several energy-efficient MAC protocols have been developed, for example, [5], [6] and [7]. These protocols aim to minimize the energy consumption of a network in different ways, but all of them employ scheduled sleeping. The idea is to avoid overhearing and idle listening. The main drawback of a periodic sleeping strategy is message latency - data have to be queued inside intermediate nodes in a multihop link until a next hop node wakes up to receive and forwards the message to the next hop. The aggregate delay can be significantly high.

As a solution to this problem, in [8] a scheduling algorithm is proposed. The algorithm requires cooperation between the network and MAC layers. The routing protocol establishes energy efficient links and the information is made available to the MAC protocol, which then determines precise on-off schedules for every node. Schedule precision means a receiving node wakes up just before a transmitting node starts to transmit. The scheduling algorithm attempts to minimize latency through a step by step waking up of intermediate nodes. By so doing, it provides a continuous data flow from a source node to a sink. The additional merit of this approach is reduced packet collision at the link layer.

In this approach, even though the network layer directly affects the MAC layer, the two layers can be developed independent from each other. For example, the routing protocol can be replaced by any other routing protocol. Moreover, while the approach promises to minimize end-to-end delay, this performance improvement is achieved by requiring a precise time synchronization strategy, which in itself is communication intensive
[9], [10]. Moreover, the algorithm does not avoid the presence of nodes near the sink node whose wake up schedule is longer than those nodes which are further away from the sink node. More importantly, since the MAC layer requires the entire routing table for calculating the transmission schedules, it would have been more meaningful to merge the two layers as one and altogether avoid the unnecessary communication overhead required to deliver an updated routing tables from the network to the link layer. A relaying architecture that is directly integrated into the physical layer is presented in [11].

\section{B. MAC-CROSS}

Suh et al. [12] propose a similar approach (called MAC-CROSS) to minimize the number of nodes in a network that stay idle unnecessarily. MAC-CROSS takes advantage of inter-layer cooperation between the link (MAC) and the network layers. As in the previous approach, routing information is directly used by the MAC protocol to maximize the sleeping duration of individual nodes. Accordingly, Sensor nodes wake up only when they belong to a routing path and their NAV ${ }^{1}$ expires. All other nodes stay asleep until another round starts. Information exchange pertaining to the routes takes place by a slight modification of the RTS/CTS packets. The "Final Destination Address" field of the RTS packet is used by the routing protocol to find the next hop address. This address is then used by the MAC protocol ("Upcoming Communication Party Address" field in a CTS packet) to put into a sleeping state all the neighbor nodes which will not participate in current data transmission. Apart from this, the routing and MAC protocols work independently.

The scheduling algorithm and MAC-CROSS are conceptually similar, but they differ in their implementation. The former is proactive (routes are established before transmission takes place) while the latter is reactive, even though routes are formed dynamically. Data transmission takes longer in MAC-CROS due to the dynamic route discovery mechanism. However, it is more reliable because a route always exists. Since end-to-end delay is not a design goal, the protocol implementation is relatively easier (it avoids the need for time synchronization). It must be remarked that disregard of endto-end transmission delay causes the data forwarding interruption problem [14]). Additionally, MAC-CROSS is not scalable, as delay increases with increasing number

\footnotetext{
${ }^{1} \mathrm{~A}$ short duration sleep schedule offered by a network allocation vector [13].
} 
of nodes - for every data transmission two hop neighbor nodes are "forced" to sleep.

\section{Coalition-Aided (CA)}

Cluster formation is one way of establishing selforganization in wireless sensor networks [15], [16]). The basic idea is to periodically elect a number of cluster-heads that satisfy certain application-specific requirements and associate child nodes with these cluster heads. Several approaches exist to ensure fair and energy efficient cluster-based self-organization. Most of these approaches, if not all, are based on cross-layer cooperation, due to a two stage cluster formation process, namely, the election of cluster heads and the association of child nodes with them.

Gao et al. [17] present a coalition-aided approach that is based on the conventional clustering techniques, but exhibits some interesting distinctions. Unlike the conventional cluster-based networks in which a cluster head performs the handful of communication tasks (coordination, aggregation, transmission), the nodes within a coalition carry out the tasks jointly. Specifically, data aggregation and coordination are performed by a coalition head, which is not usually in charge of data transmission. Data transmission is delegated to another node within the coalition.

The core idea behind coalition-aided approach is an improved cooperation between the physical, medium access control and network layers. These layers cooperate in different directions - top-down and bottom-up - to adapt to a varying wireless channel [18]. Routing is directly affected by the MAC and physical layer protocols because a transmitting node is selected based on the current link quality and the remaining energy. In other words, a transmission task is delegated to the node with the better channel conditions and more available energy. The lower layers impact the upper ones, hence the bottom-up inter-layer cooperation. Similarly, during a transmission, the signal strength is adjusted according to the link quality, hence the top-down cooperation between MAC and physical layers.

Compared to the conventional hierarchical approaches, coalition-aided design ensures efficient energy distribution among each coalition. Moreover, it improves energy utilization by dynamically adjusting the transmission signal strength. Nevertheless, the network architecture is a layered architecture and its layers are not jointly optimized, even though the cooperation between them is significantly enhanced by passing additional information.
Because of its conserved, well-defined, modular architecture, the coalition-aided approach is adaptive. The clear distinction between the layers ensures easy debugging and network maintenance. One obvious drawback is its implementation complexity. As it is, a hierarchical approach is more complex and requires intensive cooperation between nodes than mesh networks; an additional interaction between the layers introduces additional complexity. Furthermore, the inter-layer message exchange introduces additional overhead. At the same time, organizing nodes in coalitions greatly improves scalability and end-to-end transmission delay.

\section{ECPS / E2LA}

The Energy-Constrained Path Selection (ECPS) and Energy-Efficient Load Assignment (E2LA) [19] are cross-layer architectures that aim to achieve optimal energy consumption in wireless sensor networks. Both adopt the bottom-up layer cooperation strategy: lower layers pass specific control information to the upper layers that adjust their behavior accordingly. In particular, they establish a feedback from the link layer MAC to the network layer. This is the first work which strongly argues that a wireless architecture has to be MACoriented in order to provide the best performance. If the shared medium channel is time-variant, such MACadaptation strategy can be quite efficient.

The main idea of ECPS and E2LA techniques is to determine the current network state and the quality of the communication channel before an actual transmission starts. ECPS calculates the probability of sending a packet to the destination in defined numbers of transmissions for every given route, thus estimating the quality of such a route. Likewise, the E2LA algorithm takes a set of routes as an input and estimates their quality. This is done by taking the current retransmissions (collision) rates into account. All estimation results are passed to the network layer which establishes the most optimal routing scheme.

\section{INTRA-LAYER DESIGN}

In this section, three architectures are presented which can be considered as intra-layer architectures, since they move away from the notion of "painlessly" changeable or replaceable layered concept and move to a tight coupling of two or more layers which are merged or designed as an entirely new entity (layer). These architectures facilitate information flow between combined layers. Their smart coupling results in better network performance and 
efficient energy consumption. However, protocol evolution, debugging and adaptation are difficult to achieve with these architectures. Compared to the inter-layer approaches, they are error-prone and mismatch with the layers that are not included in the design might lead to unexpected network behavior and side-effect [20].

\section{A. LESOP}

The Low Energy Self-Organizing Protocol (LESOP) [21] is a cross-layer approach that entirely avoids the conventional transport and network layers. The architecture is proposed for target tracking. A node communicates with its neighbors only when it has detected a target and if the signal's energy that indicates its detection exceeds a certain threshold, $E_{t h, i}$. The receiving nodes compare the signal's energy with their own, and the node with the largest signal strength (energy) is elected as a leader (head). Upon election, the newly elected cluster head collects from the previous head node information pertaining to the target's previous location and speed, so that the target's present location and position can be estimated. All the other nodes participating in the target tracking cooperate with the new head node and send information to it.

LESOP network architecture is different from the OSI reference architecture. It consists of the physical layer, the MAC layer and the application layer. The basic assumption it makes of the physical layer as well as the limited scope of the sensing task put restriction on the reusability of the approach. LESOP requires two types of radios, a primary radio for routine wireless packets and a secondary radio to wake up sleeping nodes. Furthermore, the criteria for selecting a cluster head and forwarding packets - the target location and the associated received signal power - may not be applicable for cluster-based networks with static targets. However, analysis of the mobile object's signal strength can be thought of setting routing metrics.

\section{B. AIMRP}

The Address-Light, Integrated MAC and Routing Protocol (AIMRP) [6], merges the MAC and network layers into a single layer. AIMRP introduces a tier-based sensor network topology in which the sensor nodes organize themselves into concentric tiers around the sink and transmit data from tier to tier towards the sink. A node in the $n-t h$ tier can relay a message to the sink in $n$ hops. Due to such tier-addressing scheme, there is no need to have unique routing address. By so doing,
AIMRP attempts to reduce the cost of route discovery and maintenance overhead.

Due to the merging strategy, routing and link establishment are performed at the same time, in a single procedure. On the contrary, route discovery and link establishment are realized separately in the traditional layered architecture. Instead of the CSMA/CA control packets (RTS/CTS messages), AIMRP utilizes a modified approach that is called RTR (Ready-To-Relay) and CTR (Clear-To-Relay) control packets. These packets are useful for finding the next-hop node and to create a link. The next-hop node is chosen based on its tier identifier which has to be lower and, thus, closer to a sink. The tier identifiers are included into RTR messages which are broadcasted by a source node to the neighbors. The sensor node with a lower tier identifier responds with CTR message and becomes the next-hop node. Therefore, new Network-MAC layer performs routing and MAC functionality simultaneously.

The optimized communication between network and MAC layers conserves energy. Additionally, end-to-end communication delay is reduced because of simultaneous routing and link establishment. The cross-layer design is simple and the addressing technique is light-weight.

AIMRP's considerable energy saving and network performance is achieved under specific conditions, nevertheless. Firstly, the sink should be placed as closer to the centre of the deployment field as possible; otherwise it can cause uneven energy dissipation and earlier network partitioning. Secondly, the approach is not scalable - in order to establish the network and organize the nodes into corresponding tiers, the sink sends out special messages that arrive at the receiving nodes with varying received power. The nodes should decide to which tier they belong based on the power level of the message received. A node does not participate in the sensing task unless it directly receives a message from the sink, which implies that it should be located within the maximum transmission range of the sink node.

\section{EMI}

Due to the existence of significant differences between wired and wireless networks, refining the OSI model to meet requirements of wireless networks is not straightforward. This means that the OSI architecture has to be significantly changed in order to meet most wireless network requirements. Some of the architectures introduce an extra entity that acts as storage or a database to enable new form of information flows between the layers, thus, changing the core principle of 


\begin{tabular}{|c|c|c|c|c|c|c|c|}
\hline \multirow[t]{2}{*}{ Layers } & \multicolumn{4}{|c|}{ Inter-Layer Design } & \multicolumn{3}{|c|}{ Intra-Layer Design } \\
\hline & EES & MAC-CROSS & $\mathrm{CA}$ & ECPS/E2LA & LESOP & AIMPRP & EMI \\
\hline \multicolumn{8}{|c|}{ Application } \\
\hline \multicolumn{8}{|l|}{ Network } \\
\hline \multicolumn{8}{|l|}{ Link } \\
\hline PHY & & & & & & & \\
\hline
\end{tabular}

Fig. 1. Comparision of dependency between the layers in wireless sensor network architectures

OSI - sequential information processing by the layers. Such an architectural evolution led to a completely new architectural platform design - the Embedded Wireless Interconnect (EWI) [22].

EWI is built on two layers: the System Layer and the Wireless Link layer. The bottom Wireless Link layer supplies a library of wireless transmission modules to the upper System layer. The System layer decides the organization of the wireless links by exploiting the tradeoff between application-specific QoS gain and energy consumption. Generally speaking, EWI can be represented as a wireless 5-layer OSI stack without transport and network layers, where the application layer is a part of the System Layer; and the MAC and physical layers are parts of the Wireless Link layer. EWI proposes a new interconnection model in which modules are implemented as cross-layer services and information flows between them.

The motivation for the EMI platform is the inherent nature of wireless sensor networks. Since message exchanges in sensor networks can be characterized as event-centric, location-centric, or data-centric, packets routing is necessarily application specific. Therefore, nodes do not need to be directly interconnected with a wired infrastructure. Instead, the acquired information is serviced to data collectors (sinks) or passed through a gateway.

EWI defines three layers only to support message exchange: application, MAC and physical. This way, it attempts to reduce complexity and the additional overhead produced by the network and transport layers. However, the platform implicitly assumes that routing essentially takes place by either flooding or gossiping, and the cost of these approaches should be taken into account.

\section{Discussion}

Interest in wireless sensor networks has lead to an explosion in the number of generic and application-specific network architectures. Concern for energy-efficiency, self-organization and reliable sensing has been the driving force for many of the architectures. While these architectures take the OSI framework as a reference, they introduce new cross-layer aspects and remove or merge some of the layers of the reference architecture. For example, the transport layer is either entirely removed or merged with the routing or application layer; and the session and presentation layers are considered not to be applicable. Furthermore, there is a strong cross-layer dependency in wireless sensor network architectures, not only to enable efficient data communication, but also to achieve significant energy gain and performance adaptation.

In this paper, cross-layer dependencies are classified into inter-layer and intra-layer architectures. The classification is an attempt to avoid an overuse of the term "cross-layer". The inter-layer architectures enable vital information exchange between the layers to fine tune protocol parameters and to set route selection criteria (metrics). These architectures leave the hierarchical (layered) approach intact and support modularization. As a result, a protocol in one layer can be modified or replaced without significant impact on the protocols in the other layers as long as the modified or replacing protocol provides the same services that have been provided by the previous protocol. The intra-layer architectures merge two or more layers or introduce an entirely new network structure. In this case, protocol update or modification should take place in several layers at the same time.

The architectures presented in this paper reflect the existence of tight dependencies between the network and the link layers. This is because, nearly always, if 
a communication takes place between two nodes (pointto-point), a multi-hop communication will follow, since a remote sink is the ultimate data consumer. Surprisingly, only one of the approaches, i.e., EMI, establishes strong dependency between the application layer and the remaining lower-layers. Because wireless sensor networks are application-specific, one should expect that the application requirements such as packet arrival and error rate, end-to-end latency, and network lifetime, should be considered to configure the various protocols that manage and support routing, sleeping schedule, back-off time, contention window, transmission rate, and transmission power.

Figure 1 summarizes the seven architectures that have been discussed in this paper. It illustrates the dependency between the layers in the communication stack. The arrow direction indicates the direction of information flow (the layer to which the arrow indicates depends on information from the layer to which the tail of the arrow indicates) to tune protocol parameters.

\section{REFERENCES}

[1] Qian Dong and Waltenegus Dargie. Analysis of collision probability in unsaturated situation. In The 25th ACM Symposium On Applied Computing, 2010.

[2] V. Srivastava and M. Motani. Cross-layer design: a survey and the road ahead. IEEE communication magazine, 43(12).

[3] M.L. Sichitiu. Cross-layer scheduling for power efficiency in wireless sensor networks. In IEEE INFOCOM 2004, volume 3, pages 1740-1750, 2004.

[4] I. F. Akyildiz, W. Su, Y. Sankarasubramaniam, and E. Cayirci. survey on sensor networks. IEEE Communications Magazine, 40(8):102-114, August 2002.

[5] J. Haapola, Z. Shelby, C. Pomalaza-Raez, and P. Mahonen. gcross-layer energy analysis of multihop wireless sensor networks. In The 2nd European Workshop on Wireless Sensor Networks (EWSN '05), pages 33-44, 2005.

[6] S. Kulkarni, A. Iyer, and C. Rosenberg. An address-light, integrated mac and routing protocol for wireless sensor networks. IEEE/ACM Transactions on Networking, pages 793-806, 2006.

[7] W. Ye Li and J. Heidemann. Energy and latency control in low duty cycle mac protocols. In The 2005 IEEE Wireless Communications and Networking Conference, volume 2, pages 676-682, 2005.

[8] S. Radhakrishnan Zheng and V. Sarangan. Pmac: An adaptive energy-efficient mac protocol for wireless sensor networks. In The 19th International Parallel and Distributed Processing Symposium, 2005.
[9] Xiaojuan Chao, Waltenegus Dargie, and Guan Lin. Energy model for h2s monitoring wireless sensor network. In CSE '08: Proceedings of the 2008 11th IEEE International Conference on Computational Science and Engineering, pages 402-409, Washington, DC, USA, 2008. IEEE Computer Society.

[10] Chao Xiaojuan Dargie, Waltenegus and Mieso K. Denko. Modelling the energy cost of a fully operational wireless sensor network. Springer Journal of Telecommunication Systems, 44(1), 2010.

[11] V. Kawadia and P. R. Kumar. A cautionary perspective on cross-layer design. IEEE Wireless Communications, (1):3-11, 2005.

[12] S.D. Muruganathan, D.C.F. Ma, R.I. Bhasin, and A.O. Fapojuwo. A centralized energy-efficient routing protocol for wireless sensor networks. IEEE Radio Communication magazine, pages 8-13, 2005.

[13] W. Ye, J. Heidemann, and D. Estrin. An energy-efficient mac protocol for wireless sensor networks. In The 21st Conference of the IEEE Computer and Communications Societies (INFOCOM), volume 3, pages 1567 - 1576, 2001.

[14] Gang Lu, Bhaskar Krishnamachari, and Cauligi Raghavendra. An adaptive energy-efficient and low-latency mac for data gathering in sensor networks. In The 18th International Parallel and Distributed Processing Symposium, 2004.

[15] V.Rajendran, J.J. Garcia-Luna-Aceves, and K.Obraczka. Energy-efficient, application-aware medium access for sensor networks. In The 2nd IEEE International Conference on Mobile Ad-hoc and Sensor Systems, 2005.

[16] Vikas Kawadi and P. R. Kumar. Principles and protocols for power control in wireless ad hoc networks. IEEE journal on selected areas in communications, pages 76-88, 2005.

[17] Qinghai Gao, Junshan Zhang, Xuemin (Sherman) Shen, and Bryan Larish. A cross-layer optimization approach for energy efficient wireless sensor networks: Coalition-aided data aggregation, cooperative communication, and energy balancing. Hindawi Publishing Corporation, Advances in Multimedia, 2007.

[18] M. V. Schaar and N. S. Shankar. Cross-layer wireless multimedia transmission: Challenges, principles, and new paradigms. IEEE Wireless Communications, 12(4):50-58, 2005.

[19] A. Safwat, H. Hassanein, and H. Mouftah. Ecps and e2la: new paradigms for energy efficiency in wireless ad hoc and sensor networks. In Global Telecommunications Conference, 2003. GLOBECOM '03. IEEE, volume 06, 2003.

[20] Changsu Suh, Young-Bae Ko, and Dong-Min Son. An energy efficient cross layer mac protocol for wireless sensor networks. In APWeb 2006, pages 410-419, 2006.

[21] Liang Song and Dimitrios Hatzinakos. A cross-layer architecture of wireless sensor networks for target tracking. IEEE/ACM Trans. Netw., 15(1):145-158, 2007.

[22] Liang Song and Dimitrios Hatzinakos. Embedded wireless interconnect for sensor networks: Concept and example. In 4th IEEE conference on Consumer Communications and Networking (CCNC 2007), 2007. 